\title{
Web Application for Disaster Management
}

\author{
K. Jason ${ }^{1}$, Jishnu V K ${ }^{2}$, Remya $G^{3}$ \\ Assistant Professor,JCT College of Engineering and Technology, Coimbatore, Tamil Nadu, India ${ }^{1}$ \\ IV Year Cse, JCT College of Engineering and Technology,Coimbatore, Tamil Nadu, India ${ }^{2,3}$
}

\begin{abstract}
The web application for disaster management can provide all the helpline and it is used for public welfare and efficient management of disaster. However the existing system have some such like less accuracy and less prediction. This paper present web application for disaster management for public welfare and efficient management easily. This application include all the detail about help lines and government bodies to be contact in the stage of emergency. Then we have to upload all the news. We have all the government data set and disaster dataset. All the dataset have to analyze then those information are sort it based on user needs. Then it uploaded to this web app. GPS and hazard mapping is used to identify the areas of concern and giving the exact location along with a future prediction. This web application is developed by using HTML, CSS, Bootstrap and Python. This papers main goal is to develop a web app for disaster management. This system is very useful for at the time of disaster.
\end{abstract}

Keywords: Disaster, Hazard Mapping ,Data Set, Help Lines

\section{INTRODUCTION}

The past disaster management systems are widely existing at many places due to some disadvantages. However our web application for disaster management have a)quick prediction b)accuracy c)increased searching speed d)predict the exact location of concern areas e)user can search for the helpline at any point of time. This paper present the web app that is used for disaster management for public welfare and efficient management of disaster. Recent natural disaster have highlighted the need for disaster preparedness . planning and management. In this paper we focus on developing an ontology structure of element for web based disaster management system. This ontology structure is further coded into a web based system that allowing easy online access. People can access this app at any time at anywhere . This system have interface that provide easier communication. Whatever happened during the disaster, people can updated the information news happening around the world through the internet. Once the user logged the web app ,the user can get all the information and the system also provide feedback form for sharing users review.

\section{PROPOSED SYSTEM}

The proposed system includes various details about disasters, concern areas, helpline, government bodies, shelter homes. This web application used for accessing any information and basic needs for users at the stage of emergency. In this system we use the GPS and hazard mapping technique so we can identify the areas of concern and giving the exact location along with the future predictions .It include all the details of the helpline of the government bodies to be contacted in the stage of emergency. The software should have an add on version for shelter or rescue homes .path finding and random forest algorithm are used.HTML,CSS,PYTHON are the core language used for developing this software. The user can upload any information related to this . they can also access any information at any time by using this web application.

\section{MODULES}

\section{Administration System:}

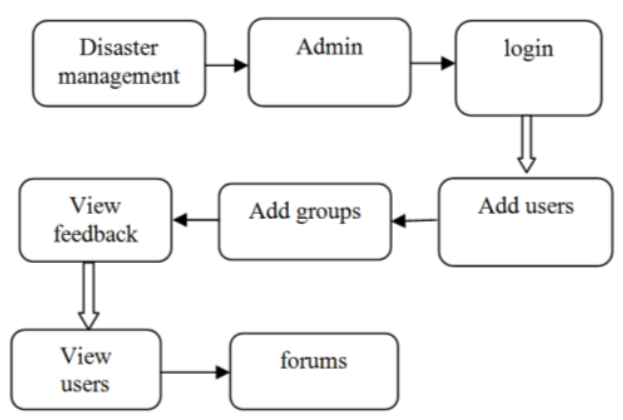




\section{IJARCCE}

Vol. 8, Issue 2, February 2019

The administration system has admin login, and database. The admin controls the entire software .the genuinity and trustworthy of the application relies on this domain. Admin will have the cross heck over each and every aspect of the app which include the database along with the client post. Updation and notifications will be send to the users on a particular interval of time.

Technical input: There will be a highly confidential login details for the admin in the first setup.

User/client system: The entire system is developed with various aspects of database and helping hands in order for the complete client satisfication. The user system has user sign up and login. Once the user logged to this system, the user can view the details and give the user feedback. Forum also included in the user system so the user will update any information regarding this .Interface between the admins can be made easy by using the datasets .

\section{TECHNICAL SETUP}

1)User registration/sign up

2) $\operatorname{login}[$ valid id is must]

3)post option[help or data notification]

4)application tracking[to end]

5)feedback options

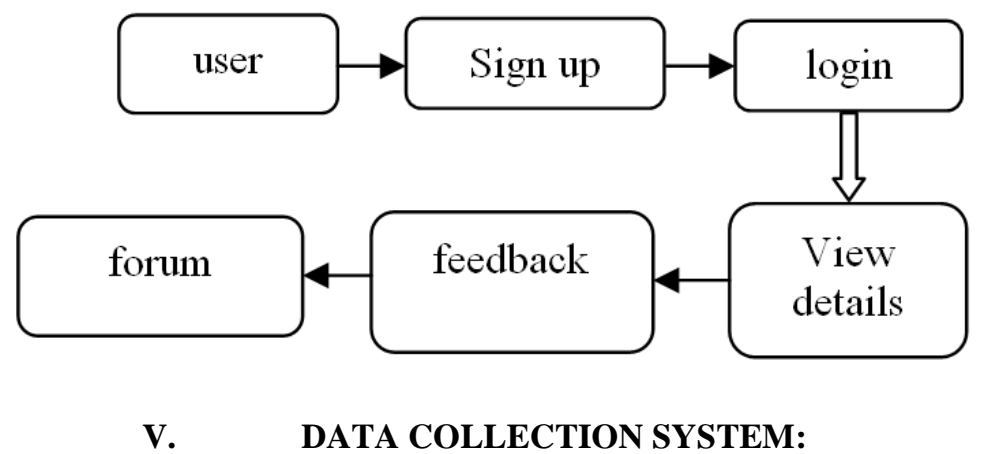

Data collection system is the core of this app and it also serve as an interface between the admin ,client ad rescue team. The data collection system include the geographical map ,hazard mapping and other data's like number of people cattles etc..in a given area along with its vegetation .climate predictions are tied up with weather forecast department which adds on the app to be all in one self dependent app. The data includes the statistical analysis of requirements volunteers ,basic needs etc.. in a particular area or rescue homes. The core sections makes the app superior over all other innovations as of a trustworthy and dependent app.

\section{Dataset:}

This include all the details of the government bodies and disasters that happened nearby .All the dataset have to analyze then those information are sorted based on the user needs .then it uploads to this website .

\section{Data Preprocessor:}

It converts quantity into numbers and used for classification.Classification and regression are involved in this process. SVM algorithm are used for the classification and regression purposes it used for analyzing data and used for the classification and regression .dresses and foods can be classified based on the priority .they can be set to used differently by the gender.

\section{Data Prediction:}

The final stage of the data set is data prediction.

It predicts the path and vulnerable area to be affected by the disaster.it uses the path finding algorithms are used for finding the shortest path.

The machine learning algorithms are used here .mostly the predictions are based on the algorithms that are used in the system. This plays a major role in the data predictions .all the algorithms are practically executed . 


\section{IJARCCE}

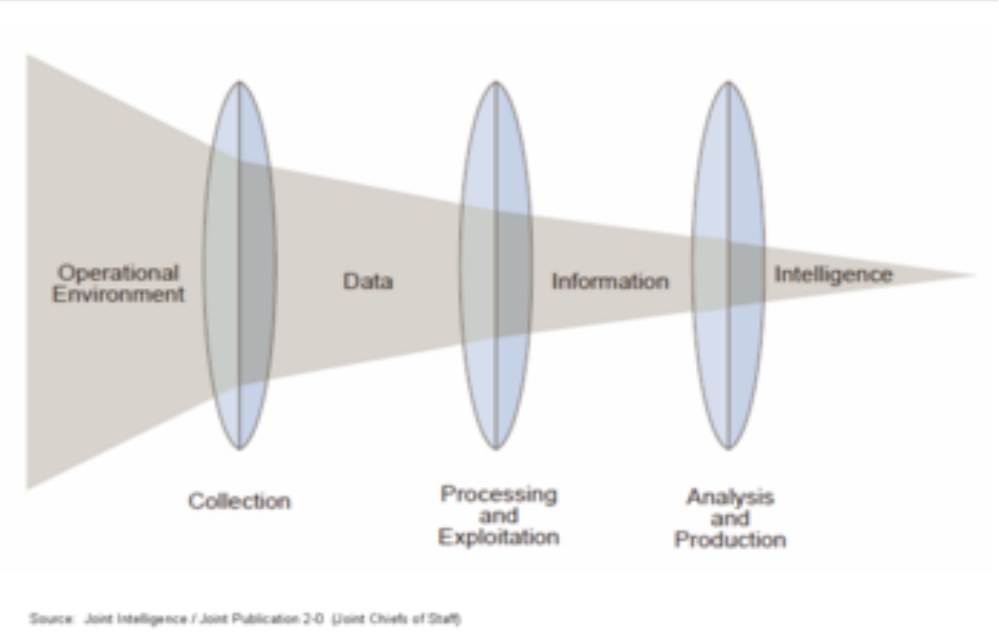

\section{Data visualization}

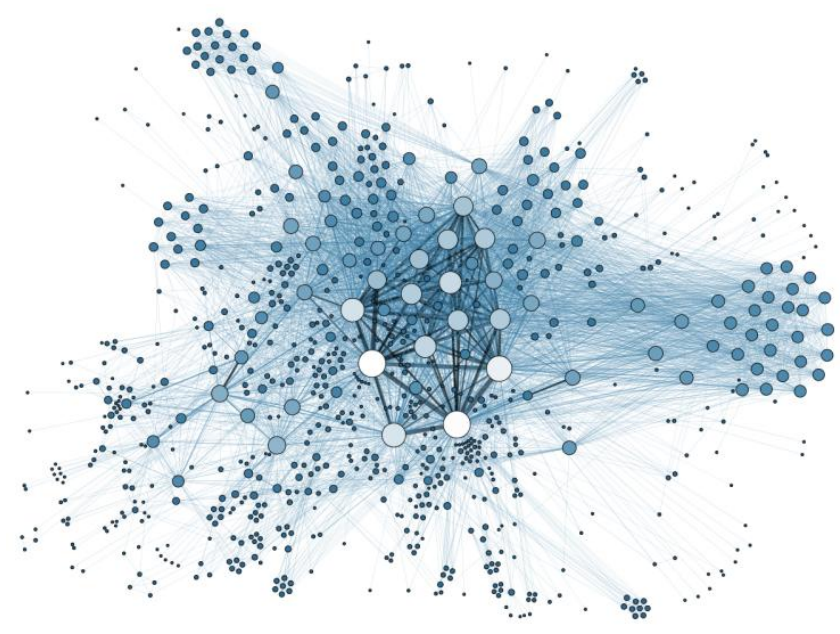

VI. ACKNOWLEDGEMENT

We would like to express our sincere gratitude to the staff of Department Of Computer Science and Engineering, JCT College Of Engineering And Technology

\section{FUTURE SCOPE}

The updations in a certain period could help the user to hold the system .though we have enough network connectivity offline mode can also be used to save data . trustworthy and genuinity is the major concern for the user and here we offer those. Notifications will help the user to understand the situation and then act accordingly .notification and updations will be send periodically to make sure that the user hangs on our systems. The new add on versions will be available to the user in a particular interval of time .once the user logged in to the web page he can get all the information and the system also provide feedback for sharing the users review .offline mode specification will be added to the system which could help the user to view or access the information anywhere at anytime

\section{CONCLUSION}

This project deals with the major technique that a web application could hold (i.e) data analytic and machine learning .the information can be easily accessed which is provided by the government this system saves time and resources when compared the existing system updates will be carried out by the admin while crosschecking information that is being noti 


\section{IJARCCE}

\section{International Journal of Advanced Research in Computer and Communication Engineering}

Vol. 8, Issue 2, February 2019

\section{REFERENCES}

[1]. K.-W. Fu and M. Chau, "Reality check for the Chinese microblog space:A random sampling approach," PLoS ONE, vol. 8, no. 3, p. e58356, Mar. 2013.

[2]. P. Fan, H. Wang, Z. Jiang, and P. Li, "Measurement of microblogging

[3]. M. Moriya and M. Ryoke, "Information balance between transmitters and receivers based on the twitter after great East Japan earthquake," Int. J. Knowl. Syst. Sci., vol. 4, no. 2, pp. 66-76, Apr. 2013.

[4]. J. Y. Jung and M. Moro, "Multi-level functionality of social media

[5]. K. Lerman and R. Ghosh, "Information contagion: An empirical study of the spread of news on digg and Twitter social networks," Comput.Sci., vol. 52, pp. 166-176, Mar. 2010

[6]. J. N. Cummings, B. Butler, and R. Kraut, "The quality of online social relationships," Commun. ACM, vol. 45, no. 7, pp.103-108, Jul. 2002, doi: $10.1145 / 514236.514242$.

[7]. A.-L. Barabási, "The origin of bursts and heavy tails in human dynamics," Nature, vol. 435, no. 7039, pp. 207-211, May 2005

[8]. E. David and K. Jon, Networks, Crowds, and Markets: Reasoning About a Highly Connected World. New York, NY, USA: Cambridge Univ. Press, 2010.

[9]. T. Antal, P. L. Krapivsky, and S. Redner, "Social balance on networks: The dynamics of friendship and hatred," Phys. D Nonlinear Phenom., vol. 224, nos. 1-2, pp. 130-136, Mar. 2006.

[10]. J. Kunegis, A. Lommatzsch, and C. Bauckhage, "The slashdot zoo: Mining a social network with negative edges," in Proc. Int. WWW Conf., Madrid, Spain, 2009, pp. 741-750

[11]. J. Leskovec, D. Huttenlocher, and J. Kleinberg, "Signed networks in social media," in Proc. CHI, ACM, Atlanta, GA, USA, 2010, pp. 13611370.

[12]. C. Chew and G. Eysenbach, "Pandemics in the age of Twitter: Content analysis of tweets during the 2009 H1N1 outbreak," PLoS ONE, vol. 5, no. 11, p. e14118, 2010. 